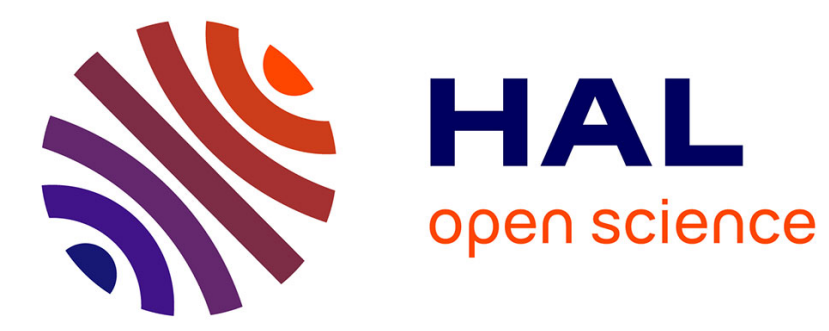

\title{
Symbolic deep learning based prognostics for dynamic operating proton exchange membrane fuel cells
}

Chu Wang, Zhongliang Li, Rachid Outbib, Manfeng Dou, Dongdong Zhao

\section{To cite this version:}

Chu Wang, Zhongliang Li, Rachid Outbib, Manfeng Dou, Dongdong Zhao. Symbolic deep learning based prognostics for dynamic operating proton exchange membrane fuel cells. Applied Energy, 2022, 305, pp.117918. 10.1016/j.apenergy.2021.117918 . hal-03362764

\section{HAL Id: hal-03362764 https://hal.science/hal-03362764}

Submitted on 3 Oct 2021

HAL is a multi-disciplinary open access archive for the deposit and dissemination of scientific research documents, whether they are published or not. The documents may come from teaching and research institutions in France or abroad, or from public or private research centers.
L'archive ouverte pluridisciplinaire $\mathbf{H A L}$, est destinée au dépôt et à la diffusion de documents scientifiques de niveau recherche, publiés ou non, émanant des établissements d'enseignement et de recherche français ou étrangers, des laboratoires publics ou privés. 


\title{
Symbolic Deep Learning Based Prognostics for Dynamic Operating Proton Exchange Membrane Fuel Cells
}

\author{
Chu Wang ${ }^{\text {a,b, }}{ }^{*}$, Zhongliang Li ${ }^{\text {b,c }}$, Rachid Outbib ${ }^{b}$, Manfeng Dou ${ }^{a}$ and Dongdong Zhao ${ }^{a}$ \\ ${ }^{\text {a }}$ School of Automation, Northwestern Polytechnical University, Xi'an 710072, China \\ ${ }^{\mathrm{b}}$ LIS Lab (UMR CNRS 7020), Aix-Marseille University, 13397 Marseille, France \\ ${ }^{\mathrm{c}}$ FEMTO-ST (CNRS 6174)/FCLAB (CNRS 3539), 90010 Belfort, France \\ *Corresponding author: Chu Wang (e-mail: chu.wang@etu.univ-amu.fr)
}

\begin{abstract}
Fuel cell (FC) is a promising alternative energy source in a wide range of applications. Due to the unsatisfactory durability performance, FC has not yet been widely used. Prognostics and health management (PHM) has been demonstrated to be an effective solution to enhance the FC durability performance by predicting FC degradation characteristics and adopting health condition based control and maintenance. As the primary task of PHM, prognostics seeks to estimate the remaining useful life (RUL) of FC as early and accurately as possible. However, when FC faces dynamic operating conditions, its degradation characteristics are often hidden in the complex system dynamic behaviors, which makes prognostics challenging. To address this issue, a hybrid prognostics approach is proposed in this paper. Specifically, the health indicator of FC is extracted using a degradation behavior model and sliding-window model identification method. Subsequently, a symbolic-based long short-term memory networks (LSTM) is used to predict the health indicator degradation trend and estimate the RUL. The experimental and simulation results show that the proposed model is able to describe the dynamic behavior of the FC stack voltage and the extracted health indicator show a significant degradation trend. Moreover, health indicator prediction and RUL estimation performance can be improved by deploying the proposed symbolic-based LSTM prognostics model. The proposed approach provides a prognostic horizon approaching $50 \%$ of the FC life-cycle, and the average relative accuracy of estimated RUL is close to $90 \%$.
\end{abstract}

Keywords - Degenerative behavior model;

Symbolic-based long short-term memory networks;

Proton exchange membrane fuel cell;

Dynamic operating conditions;

Prognostic horizon

\section{Introduction}

Fuel cells (FC) convert chemical energy directly into electrical energy with proven efficiency of 60-80\% [1-2]. In addition, when hydrogen is used as the FC fuel, zero onboard emissions can be achieved as only water is generated in FC working processes. Among different FC types, proton exchange membrane fuel cells (PEMFC) is the most widely used one thanks to its advantages, such as compact system architecture, high power density, relatively low 
start-up/running temperatures. These advantages make PEMFC one of the most promising alternatives for internal combustion engines in transportation applications. In terms of actual commercial applications, PEMFC does dominate. In 2020, PEMFC shipments exceed 53,600 units and 1,030 MW, which account respectively $65 \%$ and nearly $80 \%$ of the global total [3]. However, it cannot be ignored that the durability performance of PEMFC in most applications is still far from the target values [4-5]. In particular, in transportation applications, FC often has to face complex operating conditions, which leads to low FC durability. For instance, the durability goal of PEMFC for long-haul trucks is 25,000 hours, but in reality, it is less than half of this goal in most practical cases [1,6].

Among different solutions, prognostics and health management (PHM) aims to improve the remaining useful life (RUL) of the PEMFC by providing appropriate maintenance recommendations to defer or reduce failures. In other terms, PHM is able to continuously monitor and detect FC systems, prognosticate the future State of health ( $\mathrm{SoH})$, and draw up timely control decisions to achieve the end of FC mission [7]. As the main task of PHM, prognostics attempts to estimate the RUL of FC systems as early and accurately as possible. The PHM of PEMFC is divided into three phases: "Observe", "Analyze", and "Act" [8-9]. Prognostics is the last step of "Analyze", which is based on the comprehensive analysis of system knowledge and historical data, and it also determines the performance of operational control and maintenance decisions in "Act". Therefore Prognostics is crucial for PHM.

In general, prognostics focuses on analyzing the characteristics associated with degenerative behavior, namely health indicators (HI). A common practice for selecting HI under constant load/operating conditions is to utilize the stack voltage. In fact, as demonstrated in $[8,10,11,15,16,18]$, the stack voltage is suitable for prognostics of constant load FC. Other than stack voltage, other variables can be considered as HI in load-varying applications. In [12], Bressel et al. propose to use the resistance and limiting current as HI and estimate the proposed HI trend using Extended Kalman Filter and static FC model. The HI extraction and prediction method is demonstrated to be suitable for combined heat and power applications with slow dynamics.

In 2020 alone, more than 75\% of new FC shipments are used for transportation applications [3]. In these application scenarios, the FC stack voltage is not only affected by FC degradation level, but also dependent on time-varying operating conditions and highly dynamic loads. Using the stack voltage directly as the HI is no longer effective in the case. On the other hand, in transportation applications, the dynamic operating conditions and load changes are often operator-dependent and stochastic. This makes the estimation of the internal parameters, such as resistance, more complex and complicated. To handle the dynamic operating conditions of an FC in a transportation application, in [13] and [7], the authors Jouin et al. discussed the possibility of power and cumulative energy as HI based on the polarization curves model. However, cumulative energy often carries the nuisance of error accumulation. Lechartier et al, as in [7], proposed a model based on polarization curves and electrochemical impedance spectroscopy (EIS) for prognostics and the model owns the ability to restore the behavior of FC. Nevertheless, when deployed in practice, EIS has to be deployed when the FC is shutdown or operated with constant current for minutes, which will affect its generalization. In [14], a black-box linear parameter-varying model is used for modeling the FC dynamics, and extracting the virtual voltage as HI. However, the generalization capability of the proposed method is constrained by the black-box model. Therefore, extracting HI is fraught with challenges when deploying prognostics for PEMFC 
under complex operating conditions and dynamic loads.

Based on an extracted HI series, the process of estimating RUL in prognostics can be classified into three categories: data-driven, model-driven, and hybrid approaches [5]. Pure model-driven approaches are often accompanied by strong assumptions specific to FC technical parameters, which affect their generalization ability. Data-driven (such as [1011,15-17]) and hybrid approaches (such as [7-8,13,17-19]) have been receiving more attention. In the data-driven prognostics category, long and short-term memory networks (LSTM), in deep-learning framework, possess powerful time series prediction capabilities, and the PEMFC prognostics based on the LSTM framework have received much attention in recent years [10-11,15]. However, the raw LSTM, called Vanilla-LSTM, strongly relies on the historical data (training set) and the precise prediction of the data beyond the training data range is considered as a hard task [20]. This leads to its inability to predict the degradation trend of $\mathrm{HI}$ in the long term.

The paper aims at proposing a hybrid prognostics approach to overcome the above issues. Specifically, to extract the $\mathrm{HI}$ in dynamic and complex operating conditions, a modified FC polarization curve function is built. Based on the function, HI can then be extracted with sliding-window model identification technique. Following that, to mitigate the challenge of reliable long-term prediction and improve the performance of the LSTM-based prognostics, a symbolic representation-based LSTM is developed to predict the HI evolution trend and realize RUL estimation. The proposed hybrid prognostics approach is validated using actual FC aging experimental data acquired in dynamic operating conditions.

To summarize, the contributions of this paper are twofold:

- An HI extraction method based on modified polarization function is proposed to extract reliable $\mathrm{HI}$ in dynamic operating conditions.

- A symbolic representation-based LSTM prognostics method is proposed to improve the HI long-term prediction and RUL evaluation performance.

The rest of the paper is organized as follows. The hybrid approach, including the HI extraction method and prognostics method, is presented in Section 2. The aging experiment setting and experimental data are described in Section 3 . The

evaluation results, including the $\mathrm{HI}$ extraction and prognostics, are reported in Section 4 . Section 5 concludes the paper.

\section{Symbolic-based hybrid prognostics approach}

\section{$2.1 \quad$ FC degradation behavior model}

The factors affecting FC stack degradation are complex and are coupled with operating conditions [21]. The polarization curve is often used to characterize FC steady-state performance and thus can be used for quantifying FC degradation level. However, the FC voltage dynamic behaviors cannot be treated properly in classic polarization function. In the paper, a modified polarization function is proposed to overcome this shortcoming and make the polarization curve suitable to extract the parameters able to reflect the degradation level in dynamic operating conditions. In polarization function, FC stack voltage is expressed as [22]

$$
E_{\text {stack }}=n V_{\text {cell }}=E_{\text {ocv }}-E_{\text {act }}-E_{\text {ohm }}-E_{\text {trans }}
$$


where $E_{\text {stack }}$ is the stack voltage, $E_{\text {ocv }}$ is the stack open-circuit voltage (OCV), $n$ is the number of cells integrated in the stack, and $V_{\text {cell }}$ is the voltage of a single cell. Three main types of voltage losses affect OCV during FC operation, namely, Activation Losses $\left(E_{a c t}\right)$, Ohmic Losses $\left(E_{o h m}\right)$, and Mass Transport or Concentration Losses $\left(E_{\text {trans }}\right)$. Equations (2)-(4) quantify specifically the three voltage losses mentioned above.

$$
\begin{gathered}
E_{\text {act }}=\frac{R T}{\alpha_{0} F} \ln \left(\frac{i+i_{\text {loss }}}{i_{0}}\right) \\
E_{\text {ohm }}=r_{\text {int }} i \\
E_{\text {trans }}=-B \ln \left(1-\frac{i}{i_{L}}\right)
\end{gathered}
$$

In equations (2)-(4),

$R$ is Molar gas constant $(8.3145 \mathrm{~J} / \mathrm{mol} / \mathrm{K})$;

$F$ is Faraday constant $(96485 \mathrm{C} / \mathrm{mol})$;

$\alpha_{0}$ is the charge transfer coefficient;

$T$ is the stack thermodynamic temperature $(\mathrm{K})$;

$i$ is the stack current density $\left(\mathrm{A} / \mathrm{cm}^{2}\right)$;

$i_{\text {loss }}$ is the crossover current density $\left(\mathrm{A} / \mathrm{cm}^{2}\right)$;

$i_{0}$ is the exchange current density $\left(\mathrm{A} / \mathrm{cm}^{2}\right)$;

$r_{i n t}$ is the stack equivalent internal area-specific resistance $\left(\Omega \mathrm{cm}^{2}\right)$;

$B$ is a concentration constant $(\mathrm{V})$;

$i_{L}$ is limiting current density $\left(\mathrm{A} / \mathrm{cm}^{2}\right)$.

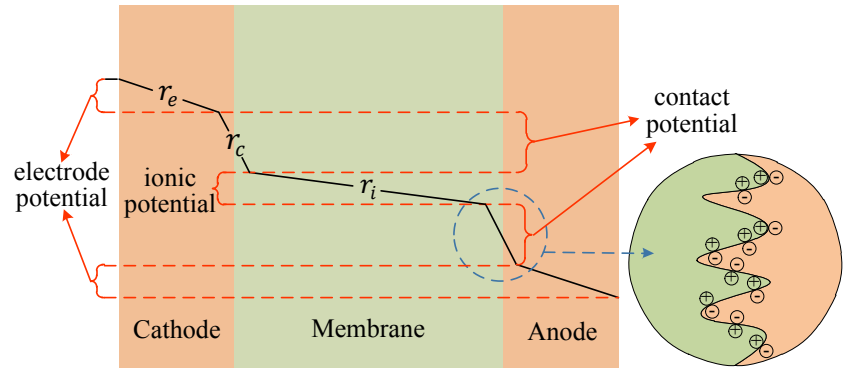

(a)

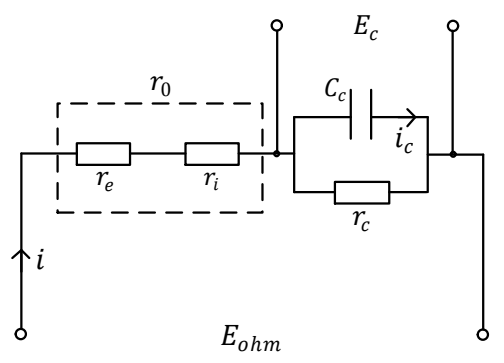

(b)

Fig. 1. The ohmic overpotential of FC, (a) correlation between various internal resistance and voltage loss; (b) equivalent circuit of internal resistance.

The internal resistance can be further decomposed into three parts, as

$$
r_{\text {int }}=r_{e}+r_{i}+r_{c}=r_{0}+r_{c}
$$

where $r_{e}$ is the electrode resistance as shown in Fig. 1 (a), the electrode resistance comes from the electrode material; $r_{i}$ is the ionic resistance comes from the proton exchange membrane; $r_{0}$ is the equivalent static resistance, which consists of $r_{e}$ and $r_{i}$ combined; $r_{c}$ is the contact resistance, which is caused mainly by the gap existing between the exchange membrane and the electrode when they are affixed. The layer of charge on or near the electrode-membrane interface is a store of electrical charge and energy, and as such behaves like an electrical capacitor [22]. One way of modeling this is by using an equivalent circuit, with the charge double layer represented by an electrical capacitor $\left(C_{c}\right)$. Fig. 1 (b) shows the equivalent circuit of the resistance within the FC. 
According to Kirchhoff's law and Ohm's law, the circuit in Fig. 1 (b) can be represented as

$$
\left\{\begin{array}{l}
E_{o h m}=r_{0} i+E_{c} \\
i_{c}=C_{c} \frac{d E_{c}}{d t} \\
E_{c}=r_{c}\left(i-i_{c}\right)
\end{array}\right.
$$

where $E_{c}$ and $i_{c}$ are the voltage and current density of the contact capacitor $C_{c}$, respectively. We can further obtain

$$
E_{\text {ohm }}+r_{c} C_{c} \frac{d E_{\text {ohm }}}{d t}=\left(r_{0}+r_{c}\right) i+r_{0} r_{c} C_{c} \frac{d i}{d t}
$$

The Laplace transform of equation (7) yields equation (8), which is further organized to obtain equation (9), and the individual variables are calculated by equation (10).

$$
\begin{gathered}
E_{\text {ohm }}(s)\left[1+\left(r_{c} C_{c}\right) s\right]=I(s)\left[\left(r_{0}+r_{c}\right)+\left(r_{0} r_{c} C_{c}\right) s\right] \\
G(s)=\frac{E_{\text {ohm }}(s)}{I(s)}=\frac{\left(r_{0} r_{c} C_{c}\right) s+\left(r_{0}+r_{c}\right)}{\left(r_{c} C_{c}\right) s+1}=\frac{p_{1} s+p_{2}}{p_{3} s+1} \\
\left\{\begin{array}{l}
r_{0}=p_{1} / p_{3} \\
r_{c}=p_{2}-p_{1} / p_{3} \\
r_{\text {int }}=p_{2} \\
C_{c}=p_{3} /\left[p_{2}-\left(p_{1} / p_{3}\right)\right]
\end{array}\right.
\end{gathered}
$$

where $G(s)$ is the system transfer function, $E_{\text {ohm }}(s)$ is the output voltage, $I(s)$ is the input current density, $s$ is the complex variable, and $p_{1}, p_{2}$, and $p_{3}$ are the system parameters to be estimated. So far, the degradation behavior model is formed by equations (1)-(5) and (10) together. In this model, $R$ and $F$ are known constants; $T, i$ and $E_{\text {stack }}$ are inputs; and the remaining parameters are outputs. In the outputs, $E_{o c v}$ and $i_{\text {loss }}$ are identified as constants, and the others will be HI candidates.

Remark: The equivalent circuit above, especially the contact capacitor, is considered to be a comprehensive equivalent for the dynamic physical processes of the fuel cell.

\subsection{Sliding-window health indicator extraction}

To achieve FC prognostics, a sliding-window health indicator extraction method is proposed. The method can be divided into three steps: sliding window procedure, parameter identification and HI selection.

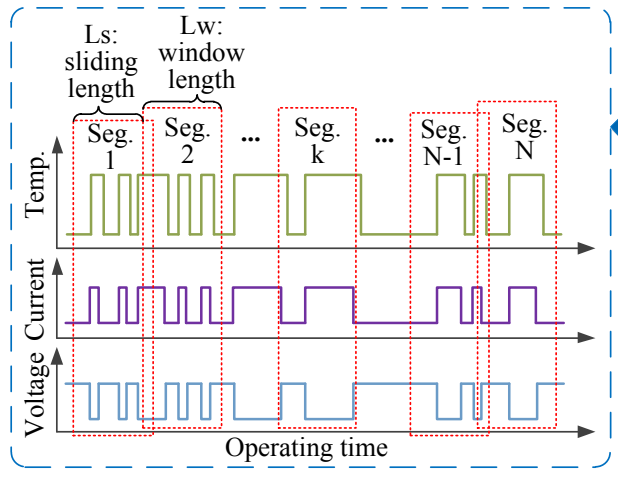

(a)

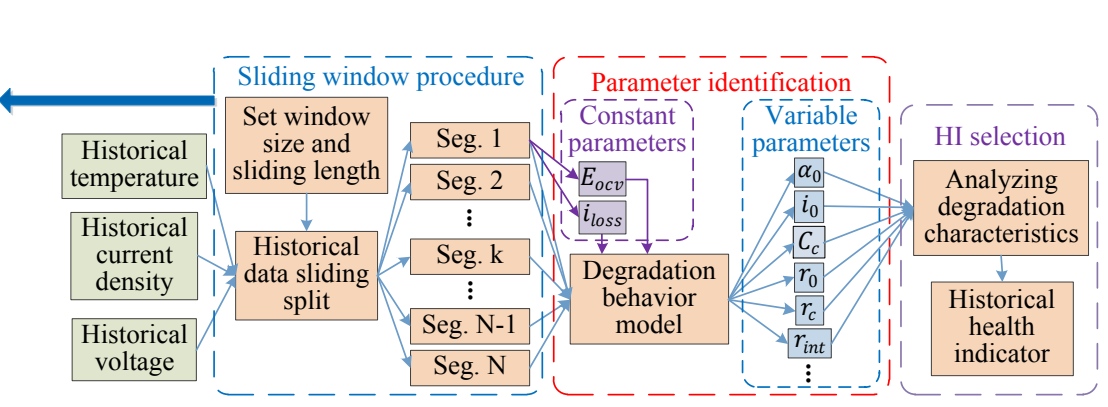

(b)

Fig. 2. Sliding-window HI extraction procedure, (a) historical data sliding-window separation; (b) parameters identification and HI selection.

As in Fig. 2(a), historical data are separated into $\mathrm{N}$ segments by sliding a data window. The window size $L_{w}$ and single sliding length $L_{s}$ are set according to the characteristics of the input historical data. The performance degradation level is considered to be unvaried in a data segment [21-22]. The window size can be set to the hourly level. Meanwhile 
the sliding length can be set to a positive value smaller than the window size. For each data segment, the Levenberg-Marquardt algorithm [23] and the simplified refined instrumental variable method for continuous-time systems (SRIVC) [24] are used to identify the parameters. Specially, $E_{\text {ocv }}$ and $i_{\text {loss }}$ are identified within first segment. Then, the segments and the identified $E_{o c v}$ and $i_{\text {loss }}$ are sequentially fed to the behavior model to identify the other parameters. Finally, HI is selected by degradation characteristics analyzing. Typically, the appropriate HI exhibits significant and consistent trends and physicochemical interpretability.

\subsection{Symbolic-based LSTM}

In the fuel cell lifetime estimation stage, classical LSTM has been successfully applied for short-term prediction of degradation trends. However, for long-term prediction, the classic LSTM trained on historical time series data (e.g., fuel cell health indicator) has limitations, such as high sensitivity to hyperparameters and the inability to break the numerical scale in the prediction stage [20]. LSTM based on symbolic representation can effectively cope with the above issues. By dimensionality reduction of time series, linear trends are extracted and maintained while accelerating the LSTM training.

\subsubsection{Symbolic-based fuel cell health indicator conversion}

The fuel cell health indicator conversion method used in this paper, called adaptive Brownian bridge-based aggregation (ABBA), allows the representation and reconstruction of a health indicator. ABBA uses increments in time and value coordinates to capture data trends in health indicator without preprocessing [25]. The implementation of the representation consists of two stages: compression and digitization.

The core of compression is to divide the $M$-dimensional health indicator $X=\left(x_{1}, x_{2}, \cdots, x_{M}\right) \in \mathbb{R}^{M}$ into $n$ segments $Q=\left(Q_{1}, Q_{2}, \cdots, Q_{n}\right) \in \mathbb{R}^{n}$. Then the compression is achieved by approximating $X$ with straight polygonal chain. To do this, ABBA selects $n+1$ breakpoints $\left(\tilde{x}_{i_{1}}=x_{1}<\tilde{x}_{i_{2}}<\cdots<\tilde{x}_{i_{n+1}}=x_{M}\right)$ in $X$. The $i_{m}$ is the time index corresponding to the $m$-th $(m=1,2, \cdots, n+1)$ breakpoint. So the $j$-th $(j=1,2, \cdots, n)$ segment can be written as $Q_{j}=\left(\tilde{x}_{i_{j}}, \tilde{x}_{i_{j}+1}, \cdots, \tilde{x}_{i_{j+1}}\right)$. In this segment, time direction increment and numerical direction increment are $\operatorname{len}_{j}=$ $i_{j+1}-i_{j}$ and $i n c_{j}=\tilde{x}_{i_{j+1}}-\tilde{x}_{i_{j}}$ respectively. Further, the squared Euclidean distance of the values in $Q_{j}$ from the polygonal chain is bounded by $\left(l e n_{j}-1\right) \cdot t o l^{2}$, as

$$
\sum_{i=i_{j}}^{i_{j+1}}(\underbrace{\tilde{x}_{i_{j}}+i n c_{j} \cdot \frac{i-i_{j}}{l e n_{j}}}_{\text {linear approximation }}-\underbrace{x_{i}}_{\begin{array}{c}
\text { actual } \\
\text { value }
\end{array}})^{2} \leq\left(l e n_{j}-1\right) \cdot t o l^{2}
$$

From the inequation, the compression rate which is represented by $l e n_{j}$ can be regulated by setting the tolerance parameter tol. Usually, this value ranges from 0.05 to 0.5 and a large tol indicates a coarser compression process [25].

Subsequently, the appropriate time and value increments form a tuple $D_{j}=\left(\operatorname{len}_{j}, i n c_{j}\right)$. Thus, the time series $X$ is represented by $n$-dimensional tuples-set $D$ as

$$
X=\left(x_{1}, x_{2}, \cdots, x_{M}\right) \Rightarrow D=\left[\left(\text { len }_{1}, \text { inc }_{1}\right),\left(\operatorname{len}_{2}, \text { inc }_{2}\right), \cdots,\left(\text { len }_{n}, \text { inc }_{n}\right)\right]
$$


Before implementing digitization, each tuple needs to be standardized and scaled. Specifically, the scaling factors $\omega_{\text {len }}$ and $\omega_{\text {inc }}$ are introduced, which represent the length and incremental weights, respectively. Both can take values between 0 and 1 and satisfy $\omega_{\text {len }}+\omega_{\text {inc }}=1$. The scaled set of tuples $\bar{D}$ can be calculated by

$$
\bar{D}=\left[\left(\omega_{\text {len }} \cdot \frac{\text { len }_{1}}{\sigma_{\text {len }}}, \omega_{\text {inc }} \cdot \frac{i n c_{1}}{\sigma_{\text {inc }}}\right),\left(\omega_{\text {len }} \cdot \frac{\text { len }_{2}}{\sigma_{\text {len }}}, \omega_{\text {inc }} \cdot \frac{i n c_{2}}{\sigma_{\text {inc }}}\right), \cdots,\left(\omega_{\text {len }} \cdot \frac{\text { len }_{n}}{\sigma_{\text {len }}}, \omega_{\text {inc }} \cdot \frac{i n c_{n}}{\sigma_{\text {inc }}}\right)\right]
$$

where $\sigma_{l e n}$ and $\sigma_{\text {inc }}$ are the standard deviation of the segment lengths and the segment increments, respectively.

The first step of digitization is to further assign $\bar{D}$ to $k$ clusters $S=\left(S_{1}, S_{2}, \cdots, S_{k}\right)$ by minimizing the within-cluster sum of squares (WCSS)

$$
\text { WCSS }=\underset{\bar{D}_{l} \in S}{\arg \min } \sum_{l=1}^{k} \sum_{\bar{D}_{l} \in S_{l}}\left\|\bar{D}_{l}-\bar{\mu}_{l}\right\|^{2}
$$

where, the $\arg \min _{x \in S} f(x)$ represent arguments $x$ for which $f(x)$ attains its smallest value. The $\|\cdot\|^{2}$ denotes the Euclidean norm, and $\bar{\mu}_{l}$ is the center of the $l$-th $(l=1,2, \cdots, k)$ cluster $S_{l}$. Further, as in equation (15), $k$ letter symbols can be used to represent each cluster separately. In practical deployments, the value of $k$ can be specified manually, as will be discussed in detail in the subsequent numerical example.

$$
\bar{D} \Rightarrow S=\left(S_{1}, S_{2}, \cdots, S_{k}\right)=\underbrace{(a, b, c, \cdots)}_{k \text { symbols }}
$$

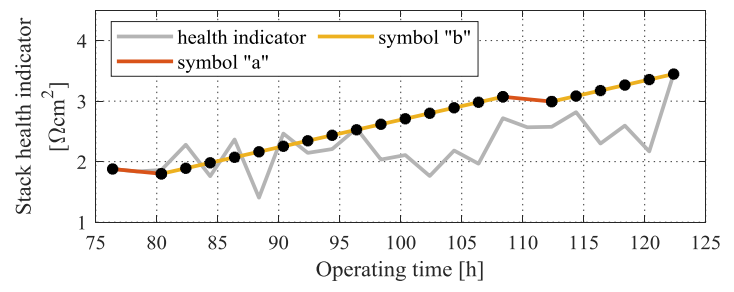

(a)

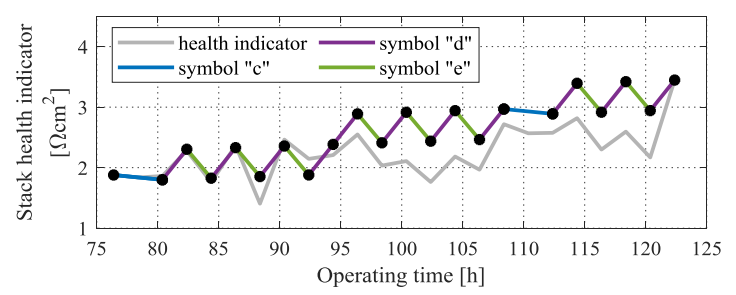

(b)

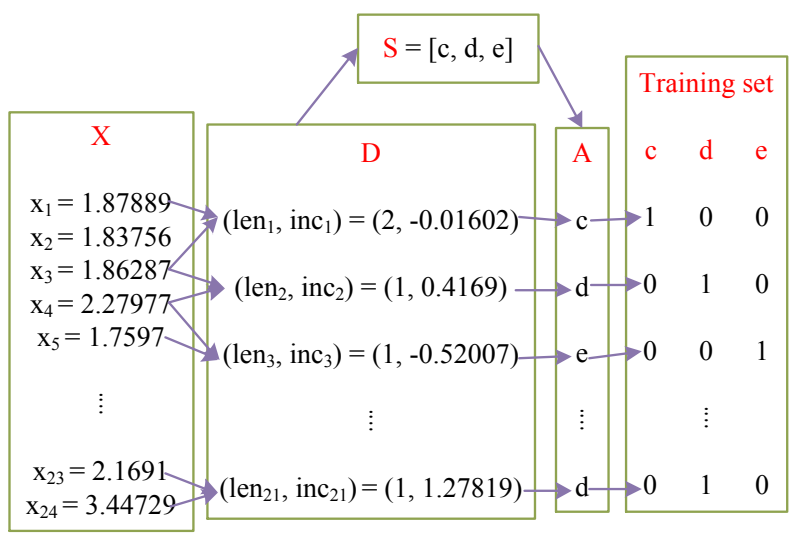

(c)

Fig. 3. Symbolic representation of the time series,

(a) setting to $k=2$ (2 symbols), the time series representation is "abbbbbbbbbbbbbbabbbbb";

(b) setting to $k=3$ ( 3 symbols), the time series representation is "cdedededdedededcdeded";

(c) the implementation of ABBA at $k=3$.

The second step of the digitization is to use the letters in $S$ to replace the tuples in the corresponding clusters in $D$ so that a string $A$ can be obtained:

$$
D \Rightarrow A=\left(a_{1}, a_{1}, \cdots, a_{n}\right)
$$

where $a_{j} \in S$. So far ABBA converts an $M$-dimensional health indicator into an $n$-letter one. The digitalized data maintains meaningful information in the original health indicator and is more lightweight, which is beneficial for the rapid deployment of a neural network in the following step. On the other hand, in contrast to the representation, the 
reconstruction means that $\mathrm{ABBA}$ converts symbolic string back to a health indicator. Reconstruction can be considered as the inverse process of representation and applies a similar mathematical approach [25].

A numerical example is shown in Fig. 3, using ABBA to represent the fuel cell health indicator. tol $=0.1$ is set in the compression phase and ABBA divides the health indicator into 21 segments. Subsequently, $\omega_{\text {len }}=\omega_{\text {inc }}=0.5$, i.e., the segment lengths and segment increments are weighted equally. Two clusters $(k=2)$ are used in Fig. 3 (a), and the obtained symbols are represented as "abbbbbbbbbbbbbbabbbbb". Then, three clusters $(k=3)$ are used in Fig. 3 (b), and the obtained symbols are represented as "cdedededdedededcdeded". Fig. 3 (c) shows the process of ABBA implementation when $k=3$, where the generated training set is used for the neural network described in subsection 2.3.2. It is seen that more detailed features can be depicted with a bigger $k$ value.

\subsubsection{ABBA-LSTM}

The LSTM is a modified recurrent neural network (RNN) that effectively solves the gradient vanishing and gradient exploding issues of RNN [26-27]. The subtlety of the LSTM is that it can assist in maintaining and updating the activity of neurons through an intrinsic chain of state transfers. As in the left part of Fig. 4, in the hidden unit of a Vanilla-LSTM (raw LSTM), there exist input gate $\left(i_{t} \in \mathbb{R}^{h}\right)$, forget gate $\left(f_{t} \in \mathbb{R}^{h}\right)$, and output gate $\left(o_{t} \in \mathbb{R}^{h}\right)$ consisting of a sigmoid function $(\sigma)$, in addition to an internal state unit $\left(\tilde{c}_{t} \in \mathbb{R}^{h}\right)$ consisting of tanh function. These can be deployed by equations (17)-(20), where $h$ denotes the number of hidden units in an LSTM layer. The output vector $\left(h_{t} \in \mathbb{R}^{h}\right)$ and the unit state $\left(c_{t} \in \mathbb{R}^{h}\right)$, which are continuously passed between each hidden unit, are represented by equations (21) and (22), respectively. It is with the collaboration of $h_{t}$ and $c_{t}$ that a special chain is formed between the individual hidden units. The mathematical realization of a LSTM unit can be summarized as

$$
\begin{gathered}
f_{t}=\sigma\left(W_{f} x_{t}+U_{f} h_{t-1}+b_{f}\right) \\
i_{t}=\sigma\left(W_{i} x_{t}+U_{i} h_{t-1}+b_{i}\right) \\
o_{t}=\sigma\left(W_{o} x_{t}+U_{o} h_{t-1}+b_{o}\right) \\
\tilde{c}_{t}=\tanh \left(W_{c} x_{t}+U_{c} h_{t-1}+b_{c}\right) \\
c_{t}=f_{t} \circ c_{t-1}+i_{t} \circ \tilde{c}_{t} \\
h_{t}=o_{t} \circ \tanh \left(c_{t}\right)
\end{gathered}
$$

where $x_{t} \in \mathbb{R}^{k}$ represents the $k$-dimensional input, and $W_{f}, W_{i}, W_{o}, W_{c} \in \mathbb{R}^{h \times k}$ represent the weight matrices of the forget gate, the input gate, the output gate and the internal state unit, respectively. $U_{f}, U_{i}, U_{o}, U_{c} \in \mathbb{R}^{h \times k}$ represent the recurrent connection matrices in the forget gate, the input gate, the output gate and the internal state unit, respectively. The operator "o" represents the Hadamard product of the elements.

However, Vanilla-LSTM trained based on the historical values of the data can hardly predict results beyond the value range of training set [20]. This constrains the direct use of Vanilla-LSTM for some specific applications, especially for fuel cell prognostics where the predicted health indicator are supposed to be in different value interval from the training data. Using time series incremental information in the ABBA symbolic representation facilitates health indicator 
evolution trend capture, which promisingly gives ABBA-LSTM the ability to predict beyond the value range limits of the original training in fuel cell prognostics.

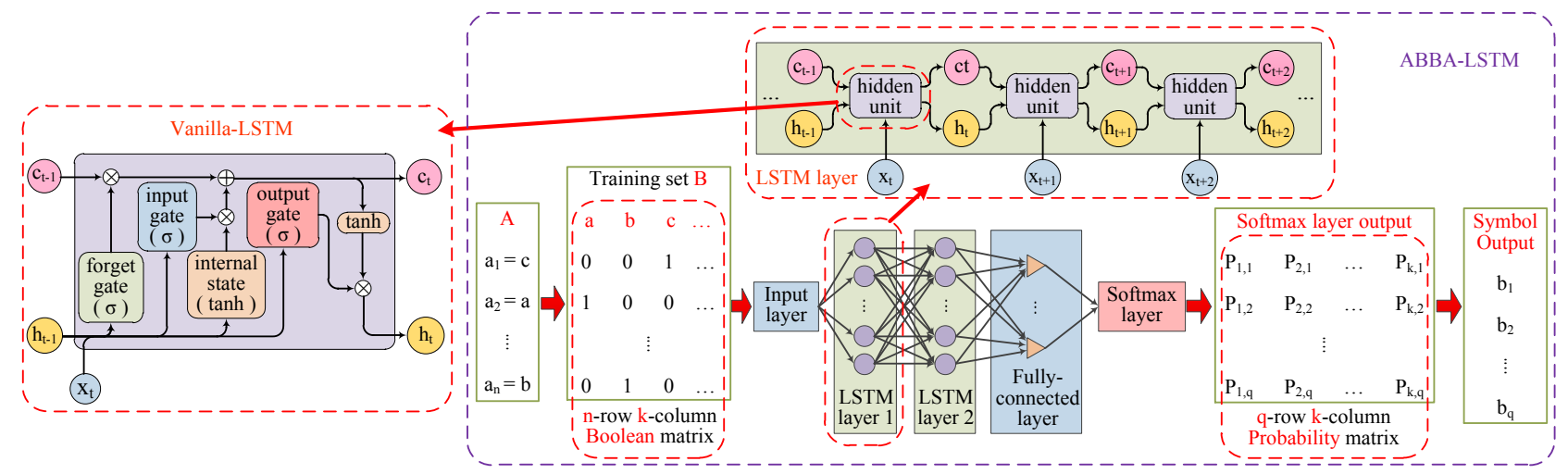

Fig. 4. The typical ABBA-LSTM structure.

The structure of ABBA-LSTM is shown in Fig. 4, which contains five layers: one input layer, two LSTM layers, one fully connected layer and one output layer. Specifically, the ABBA-LSTM input is an $n$-row $k$-column Boolean matrix $B \in \mathbb{B}^{n \times k}$. Consistent with the subsection $2.3 .1, n$ represents the number of compressed segments and $k$ represents the number of digitized symbols (number of clusters). The $j$-th $(j \in(1,2, \ldots, n))$ segment can be represented as a $k$-dimensional Boolean row vector $\left(B_{j, 1}, B_{j, 2}, \cdots, B_{j, k}\right)$. In this row vector, there is only one element corresponding to the segment symbol $a_{j}$, set as $B_{j, l}=1,(l \in(1,2, \ldots, k))$ and the rest of the elements are set to 0 . Subsequently, the input will pass through two LSTM layers, each containing 50 hidden units. Following the LSTM layers is a fully connected layer containing $k$ plain neurons (with the equivalent function $f(x)=x$ ). Finally, a softmax layer outputs a $q$-row $k$-column probability matrix, where $q$ is the number of predicted time steps. The highest probability in each row of the probability matrix is selected and its corresponding symbol is used as the output, and a string $\left(b_{1}, b_{2}, \cdots, b_{q}\right)$ can be obtained as the prediction result. This prediction result, as final output, will be converted into a health indicator by the ABBA reconstruction.

\subsection{Hybrid prognostics approach}

The hybrid prognostic approach combining the HI extraction and the ABBA-LSTM prognostic model is shown in Fig. 5.

As mentioned in Subsection 2.2, HI is identified by using the proposed the degradation behavior model and the sliding window model identification procedure. Thanks to the low preprocessing requirements of the ABBA-LSTM model, the historical HI time series can be fed directly to the model as input, as in Fig. 5 (a).In this work, to cope with the model uncertainty, multiple ABBA-LSTM models are constructed by setting $n$ random initial weight matrices. Once the models are properly trained, ABBA-LSTM models can realize one-step or few-step ahead prediction. The long-term HI prediction is achieved by iterating the prediction procedure using the last-step prediction result as input. The predicted trends are further used to estimate the RULs, as in Fig. 5 (b). The prognostic starting point corresponds to the time $t_{p}$. Given a failure threshold for HI, its intersection time with each predicted trend is the predicted end-of-life (EOL). The estimated RUL at $t_{p}$ is calculated as 


$$
R U L_{i}=E O L_{i}-t_{p}
$$

where the subscript $i$ denotes the index of $(i=1,2, \ldots, n)$. The $n$ RULs further constitute the prediction distribution. The highest peak of the distribution (not the mean) is chosen as the final RUL.

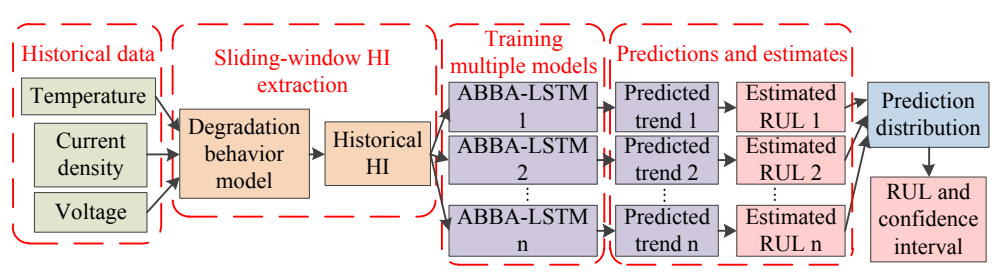

(a)

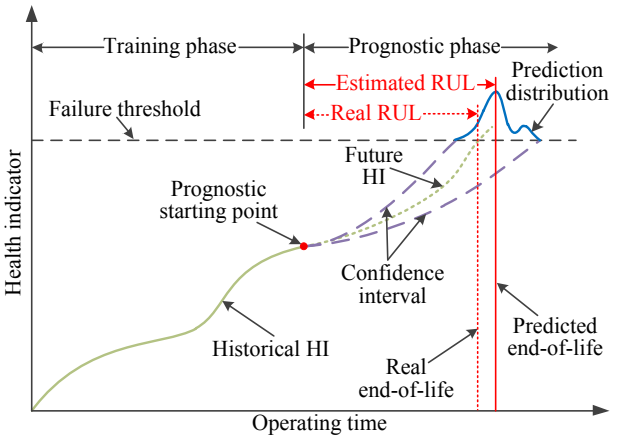

(b)

Fig. 5. Hybrid prognostic approach, (a) the implementation block diagram; (b) the remaining useful life estimation.

\section{$3 \quad$ FC aging experiments under dynamic operating conditions}

Generally, obtaining long-term lifetime data for fuel cell operation, especially under dynamic operating conditions, is fraught with challenges. A compact low-power scale FC stack with an open cathode and dead-end anode structure is focused on and used to test the proposed prognostics approach. The FC is designed with self-humidifying capability. The specific technical parameters are summarized in Table 1. The stack is integrated with a 24V DC fan for oxygen supply and cooling. The stack operating temperature is controlled by regulating the air fan and the reference varies with temperature as

$$
T_{\text {opt }}=2.5074 I+30.3585
$$

where $T_{o p t}$ is the temperature control reference and $I$ is the operating current. In the concerned compact fuel cell system, stack temperature, current and stack voltage are measured and analyzed for prognostics. It is noted that, an identical air fan is used for both air supply and temperature regulation. The air fan speed is regulated to make the fuel cell temperature attain the predefined temperature. The air pressure and stoichiometry are therefore highly coupled to temperature. Hence, the temperature is a representative variable to describe the fuel cell dynamic behavior. More details about the experimental setting and the experimental dataset can be found in [14].

Table 1 Aging Experiment Operating Condition

\begin{tabular}{ll}
\hline \hline \multicolumn{1}{c}{ FC type } & Open cathode/Dead-end anode \\
\hline Active surface & $33.63 \mathrm{~cm}^{2}$ \\
Nominal pressure at hydrogen inlet & $1.35 \mathrm{bar}$ \\
Nominal pressure at oxygen inlet & normal atmospheric pressure \\
Number of cells & 15 \\
Nominal output power & $73.5 \mathrm{~W}$ \\
Operating temperature & corresponding to current \\
Maximum temperature & $75^{\circ} \mathrm{C}$ \\
Maximum current & $13.45 \mathrm{~A}$ \\
Lowest permitted stack voltage & $7.5 \mathrm{~V}$ \\
\hline \hline
\end{tabular}

This aging experiment lasted 1486 hours, and the vast majority of the load current densities are below $0.4 \mathrm{~A} / \mathrm{cm}^{2}$, as in Fig. 6. When the FC is operated at a small current density, it suffers from little concentration loss [22]. Therefore, we 
assume that the concentration loss is combined with the linear ohmic resistance losses at low current density conditions. Further, the degradation behavior model in this case is simplified to

$$
E_{\text {stack }}=E_{\text {ocv }}-\frac{R T}{\alpha_{0} F} \ln \left(\frac{i+i_{\text {loss }}}{i_{0}}\right)-r_{\text {int }} i
$$

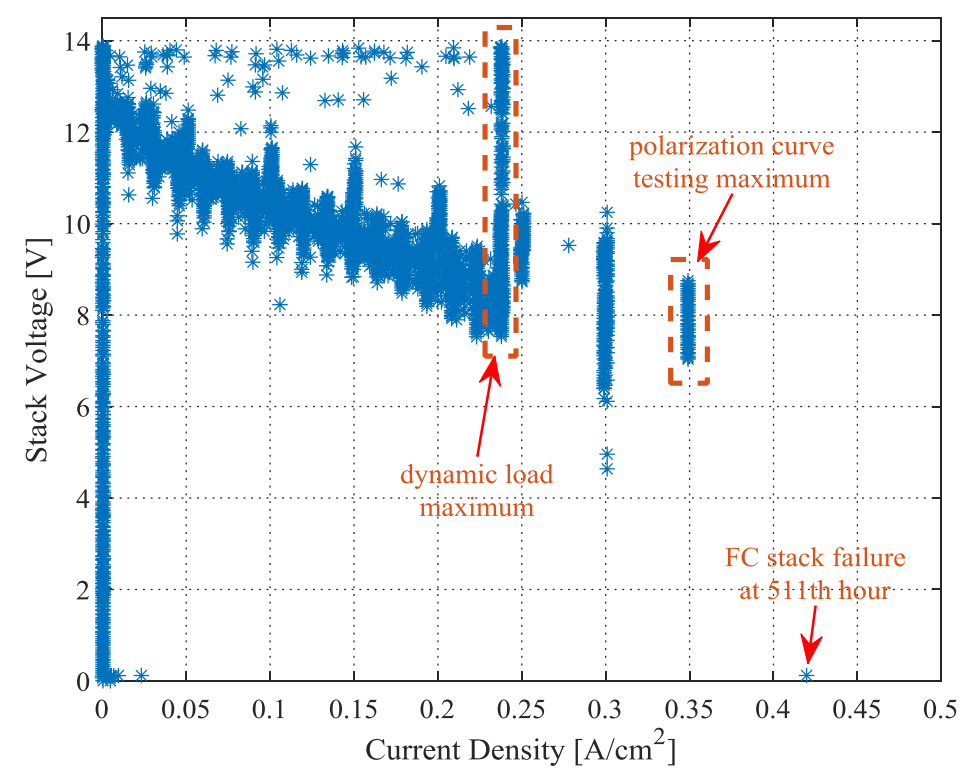

Fig. 6. Voltage-current density Curve from the 1486 hours FC aging experiment.

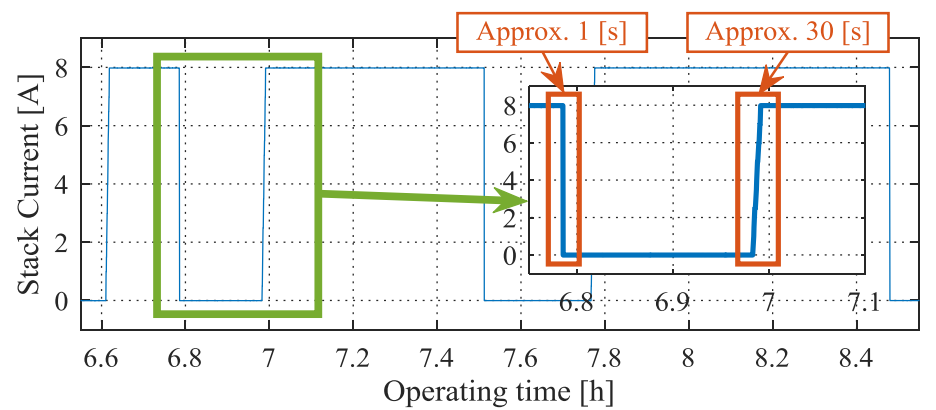

(a)

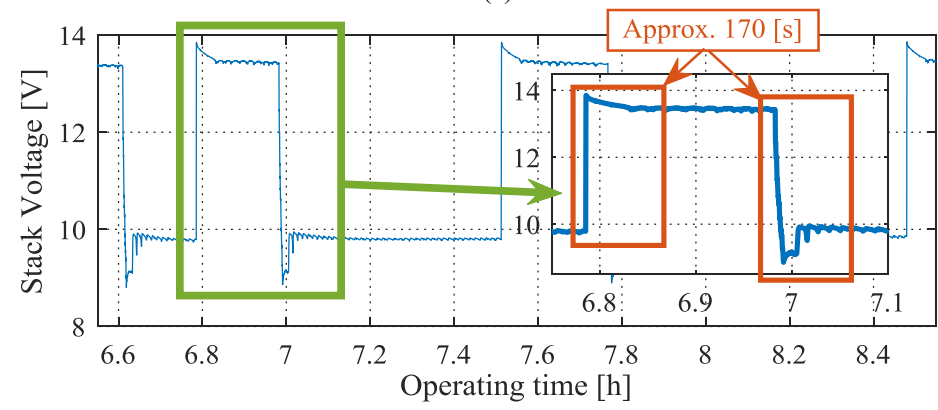

(b)

Fig. 7. Cyclical dynamics of the load: (a) operating current; (b) operating voltage.

\subsection{FC cyclical dynamics}

The aging experiments aimed to restore the dynamic operating conditions of FC in a hybrid electric vehicle (HEV). In the application, the FC stack coupled to lithium-ion batteries and functions as a range extender. Specifically, once the battery state of charge (SOC) drops to a preset threshold, the FC is activated to charge the battery with constant 
current. When the battery SOC reaches at the targeted level, the charging process is paused, and the FC system is kept as a standby. In this work, a programmable electronic load is used to realize the above mentioned cyclic start-up-standby process as Fig. 7. The operating current decreases from 8 A to 0 A for about 1 second, as in Fig. 7 (a). Correspondingly, the voltage rise process lasts about 170 seconds, as in Fig. 7 (b). On the other hand, it takes about 30 seconds to raise the operating current from $0 \mathrm{~A}$ to $8 \mathrm{~A}$, and the corresponding voltage drop process takes about 170 seconds. It is noteworthy that the figure shows the early stage of the FC stack aging experiment. As the aging experiment progresses, the operating voltage response time is slightly extended. The overshoot phenomena exhibited by the operating voltage during current transitions can be simulated and equated by a capacitor-like dynamic element as mentioned in Section 2 .

\subsection{Long-term evolution of operating conditions}

In the aging experiment, the $\mathrm{FC}$ even has to cope with the long-term evolution of operating conditions. It is important to note that, unlike the cyclic dynamic loading mentioned in subsection 3.1 , the long-term evolution of the operating conditions comes from the coupled influence of multi-scale factors. In other words, this long-term evolution reverts to a certain extent to the HEV diverse environmental effects. The Fig. 8 shows the long-term evolution of the voltage (e.g., (a), (b)) and current (e.g., (c), (d)) in the FC aging experiment. First, in order to better describe the long-term evolution of the operating conditions that occurred in this aging experiment, four characteristic time points were set: $122 \mathrm{~h}$ ( 1 ), $511 \mathrm{~h}(2), 702 \mathrm{~h}(3)$, and $168 \mathrm{~h}$ ( 4 ). The locations where the polarization curve tests were performed are marked in Fig. 8 (c), which corresponds to the anomalies that appear every approximately 168 hours in the operational behavior. The aging experimental process is then divided into four phases:

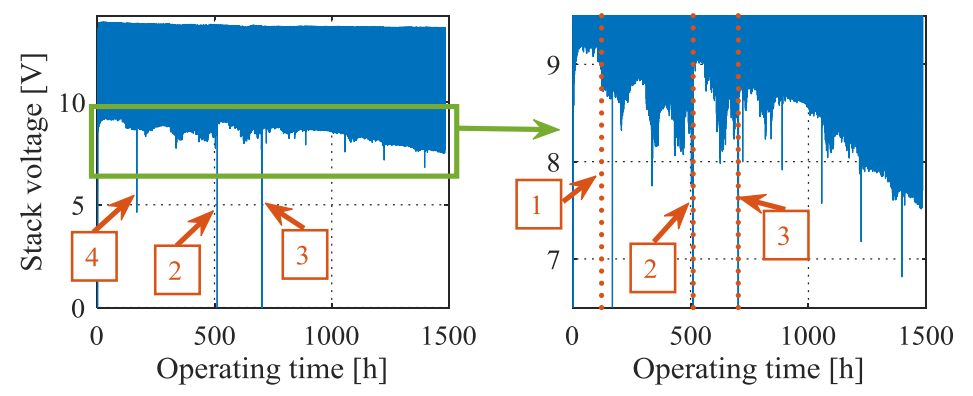

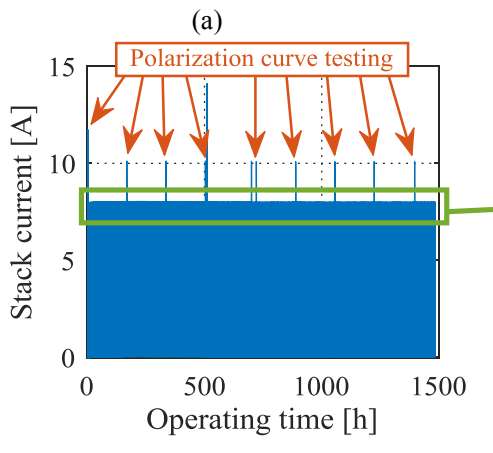

(c)

(b)

Fig. 8. Evolution of operating conditions, (a) operating voltage; (b) operating voltage in detail; (c) operating current; (d) operating current in detail; 
Phase (1), as in Fig. 8(d), is from hour 0 to point 1 . The FC stack gradually reaches the optimal working condition after the first start, influenced by the internal catalyst activity and other factors.

Phase (2), from point 1 to point 2 . At the beginning of the 2 nd test (hour 168.5), a peripheral fault occurred, represented by point 4 , which caused a sudden drop in the stack voltage but did not cause a FC shutdown. After the 4th polarization test, an abnormal FC shutdown occurred at point 2 .

Phase (3), points 2 to 3 . During the 5 th polarization test, the abnormal FC shutdown shown at point 3 occurred.

Phase (4), the remaining part after point 3. During this phase, the FC effective operating current remained essentially 8 A. After point 3 , the stack was restarted and the incomplete 5th polarization test was repeated at hour 721 .

It is worth noting that all the three stack serious faults (points 2 , 3, and 4) occurred while the polarization test was being performed or shortly after the test completed. Distinct from the load cycle variations mentioned in subsection 3.1 , the 2nd-9th polarization curve tests require the consideration of eight different currents between 0 and 10 A. This means that the operating conditions of the stack during the polarization test are more complex and prone to induce faults. In addition, the effect of ambient temperature should not be ignored. During the aging experiments between 0 hours and point 3 , occasional laboratory air conditioning system faults triggered ambient temperature fluctuations. The stack-integrated DC fan regulated the temperature while also changing the oxygen supply to the cathode, which in turn affected the operating behavior of the stack. After the FC restart at point 3 , the laboratory ambient temperature remained constant and the operating current became smooth thereafter. Correspondingly, the operating voltage after point 3 also shows a smoother reduction behavior.

\section{Performance assessment and results analysis}

\subsection{Assessment metric}

In order to fully and appropriately evaluate the proposed approach, the performance of the degenerate behavior model and the RUL estimation are validated separately in this paper. Specifically, considering that the degenerate behavior model output is identified stack voltage, the relative accuracy $\left(R A_{H I}\right)$ achieved when extracting $\mathrm{HI}$ is evaluated by

$$
R A_{H I}=\left(1-\frac{\left|E_{\text {stack }}-E_{\text {est }}\right|}{V_{\text {stack }}}\right) \times 100 \%
$$

where $E_{\text {est }}$ is the identified stack voltage.

On the other hand, the performance of RUL estimation is evaluated from 3 aspects. The first is the prognostic horizon (PH) [7,28]. In simple terms, $\mathrm{PH}$ will be given an acceptable error zone $\left(A E Z_{P H}\right)$. By setting the upper and lower boundaries parallel to the actual RUL, the $A E Z_{P H}$ is defined as the enclosing region formed by the upper and lower boundaries together with the two coordinate axes, as

$$
R U L-\alpha^{-} \times E O L \leq A E Z_{P H} \leq R U L+\alpha^{+} \times E O L
$$

where $E O L$ is the actual end of life. $\alpha^{-}$and $\alpha^{+}$are accuracy modifiers that restrict the lower and upper bounds of $A E Z_{P H}$, respectively, which in turn affect the tolerance of PH. For the FC prognostic, when the estimated RUL is biased, the result smaller than the actual RUL is usually more acceptable. On the contrary, when the estimated RUL is 
larger, it tends to lead maintenance plan lag, even the fatal failure early occurrence. In view of this, different upper and lower bounds are set, i.e., $\alpha^{+}=0.1$ and $\alpha^{-}=0.2$, respectively. If an estimated RUL can fall into $A E Z_{P H}$, the prognostic is considered reliable. When there is a breakpoint after which all estimated RULs are guaranteed to fall into $A E Z_{P H}$. Then this point is defined as the starting time point $P_{0}$, and at this time $\mathrm{PH}$ can be calculated by

$$
P H=E O L-P_{0}
$$

It is important to note that $\mathrm{PH}$ appears to be relatively lenient and it is a minimal evaluation metric. In general, for a prognostic approach, a longer PH means better performance. On the contrary, approaches that do not meet the $\mathrm{PH}$ metrics are not worth adopting.

Similar to $A E Z_{P H}$, a more stringent acceptable error zone $\left(A E Z_{\alpha-\lambda}\right)$ is introduced. Parameter $\lambda$, called window modifier is used to describe the position of the fatal failure in the PH [28]. In this paper, $\lambda$ is considered to be set to 1 , i.e., the fatal failure occurs at the EOL. $A E Z_{\alpha-\lambda}$ can be calculated by

$$
\left(1-\alpha^{-}\right) \times R U L \leq A E Z_{\alpha-\lambda} \leq\left(1-\alpha^{+}\right) \times R U L
$$

The reliability of the prognostic approach can be further assessed by examining how well the estimated RUL falls into $A E Z_{\alpha-\lambda}$, which namely $\alpha-\lambda$ performance.

Finally, in order to quantitatively assess the accuracy of the estimated RUL, the relative accuracy $\left(R A_{R U L}\right)$ is also introduced, similar to $R A_{H I}$, and can be calculated by

$$
R A_{R U L}=\left(1-\frac{\left|R U L-R U L_{\text {est }}\right|}{R U L}\right) \times 100 \%
$$

where $R U L_{e s t}$ is the estimated RUL.

\subsection{Performance of the degeneration behavior model}

The first step in deploying the hybrid prognostic approach is the sliding-window HI extraction. Specifically, the window size and sliding length are set to 2 hours. Sliding split is performed on historical data lasting 1486 hours, and parameters are identified in each window.

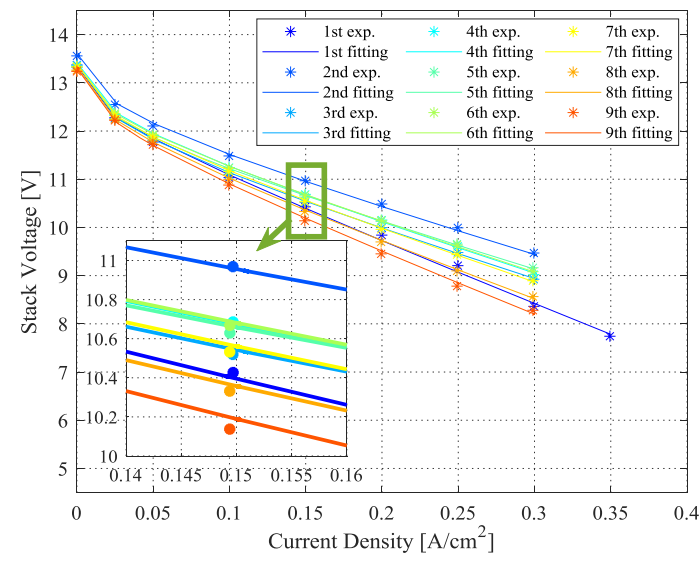

(a)
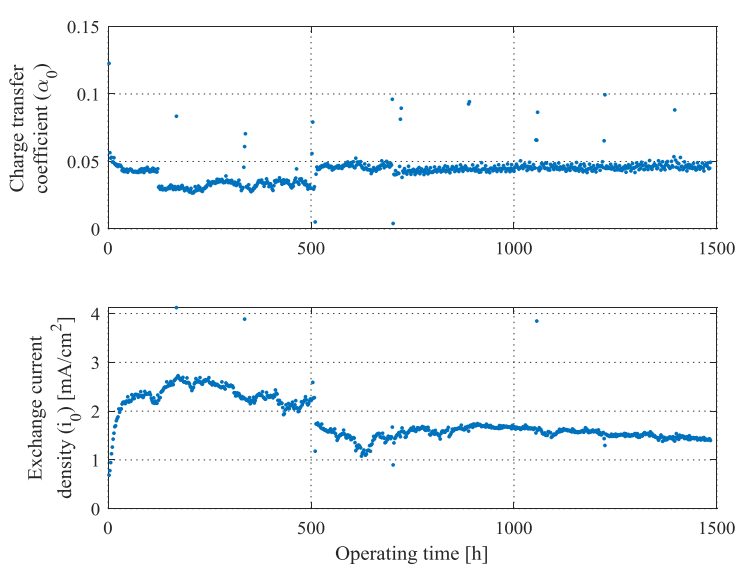

(b)

Fig. 9. Polarization test and activation losses parameters identification, (a) the 1st-9th polarization curves fitting; (b) charge transfer coefficient and exchange current density.

The polarization curves fitted by the degradation behavior model are shown in Fig. 9 (a), and the fitted results match well with the experimental data. The 2 nd test was affected by a peripheral device fault (point 4 ) and presented very 
different results from the other tests. The remaining 8 tests showed an extremely strong correlation with ohmic overpotential. Among them, the 1st, 3rd and 4th polarization curves show an increasing trend, which is very interesting and comes from the change in operating conditions and the influence of ambient temperature mentioned in Section 3 . The 4th-6th polarization curves almost overlap, which is due to the effect of fault points 2 and 3 , and in fact, a reversible performance improvement occurred when the FC is restarted after experiencing a shutdown. The 6th-9th polarization curves show a steady trend of degradation (decline).

The parameter identification procedure first determined the $E_{o c v}$ and $i_{\text {loss }}$ as in Table 2. Subsequently, as in Fig. 9 (b), the identified activation losses parameters $\alpha_{0}$ and $i_{0}$ show significant changes at points 1 , 2, and 3 , and become stabilize after 702 hours. This indicates that these two parameters are sensitive to the operation changes of FC.

Table 2 Model Parameters Identification

\begin{tabular}{cccc}
\hline \hline Parameter & Value (or range) & Unit & Note \\
\hline$E_{\text {ocv }}$ & 13.37 & $\mathrm{~V}$ & $\begin{array}{c}\text { Identified in first } \\
\text { sliding-window }\end{array}$ \\
$i_{\text {loss }}$ & 1 & $\mathrm{~mA} / \mathrm{cm}^{2}$ & Fig. 9 (b) \\
$\alpha_{0}$ & 0.004 to 0.13 & - & Fig. 10 (a) \\
$i_{0}$ & 0.6 to 4 & $\mathrm{~mA} / \mathrm{cm}^{2}$ & Fig. 10 (b) \\
$r_{0}$ & -7 to 14 & $\Omega \mathrm{cm}^{2}$ & Fig. 10 (c) \\
$r_{C}$ & -1.2 to 8 & $\Omega \mathrm{cm}^{2}$ & Fig. $10(\mathrm{~d})$ \\
$r_{\text {int }}(\mathrm{HI})$ & -3.5 to 2.5 & $\Omega \mathrm{cm}^{2}$ & \\
$C_{C}$ & 0 to 34 & $\mathrm{mF} / \mathrm{cm}^{2}$ & \\
\hline \hline
\end{tabular}

The identified Ohmic Losses parameters are shown in Fig. 10 and Table 2. Among them, $r_{c}$ and $C_{c}$ are relatively stable in the first half, while in the second half there are monotonic-like variations. Meanwhile $r_{0}$ and $r_{\text {int }}$ show a clear upward trend, and the $r_{i n t}$ represents whole stack being selected as HI. It is worth noting that some extracted resistances show negative values, which occur mainly before the 6th polarization test, especially between points 1 and 2. This corresponds to the previous analysis of the activation losses parameters $\alpha_{0}$ and $i_{0}$, indicating that the internal resistance values are also sensitive to the operating condition changes. Even so, the extracted HI still provides trend features sufficient for prognostic purposes. Further, in Fig. 10 (e), by comparing with the stack power.

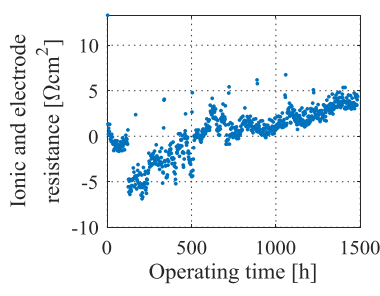

(a)

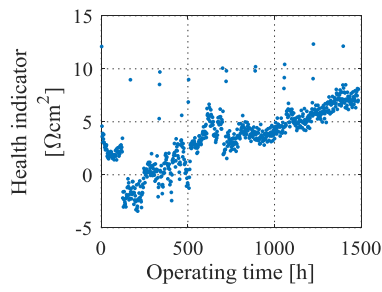

(c)

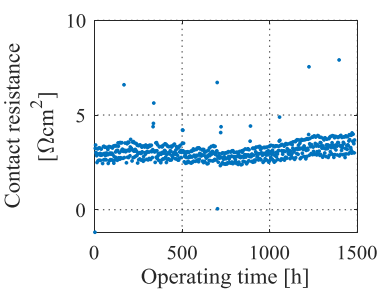

(b)

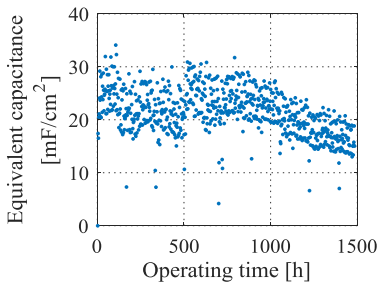

(d)
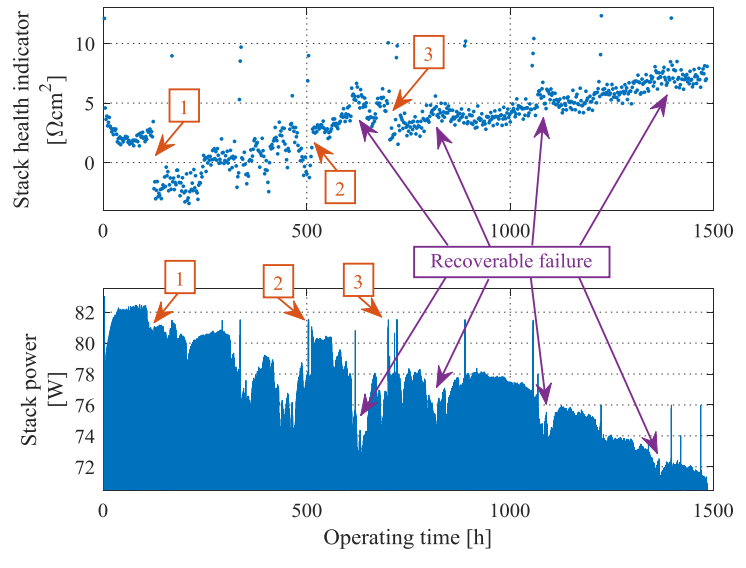

(e)

Fig. 10. Ohmic Losses parameters identification, (a) ionic and electrode resistance; (b) contact resistance; (c) health indicator (equivalent internal resistance); (d) equivalent capacitance. (e) Indication of dynamic operating conditions and faults via HI. 
The extracted HI not only shows changes in operating conditions, but also indicates faults of the stack. Specifically, when there are significant breaks or gouges in the HI, such as points 1 , 2, and 3 , it means that a change in operating conditions or a serious fault/shutdown has occurred at that time. When the HI shows a short term (about tens of hours) bump followed by a return to the original trend of change, this indicates a recoverable stack fault.

Remark: 1) The faults indicated by HI refer to abnormal behaviors of the fuel cell system, such as abnormal operation, peripheral device fault, abnormal shutdown, recoverable fault, etc. 2) the complete polarization curve test is not mandatory in $\mathrm{HI}$ extraction procedure. Nevertheless, it is recommended to do the initial polarization curve test to correctly identify the constant parameters and the reasonable parameter intervals in Table 2.

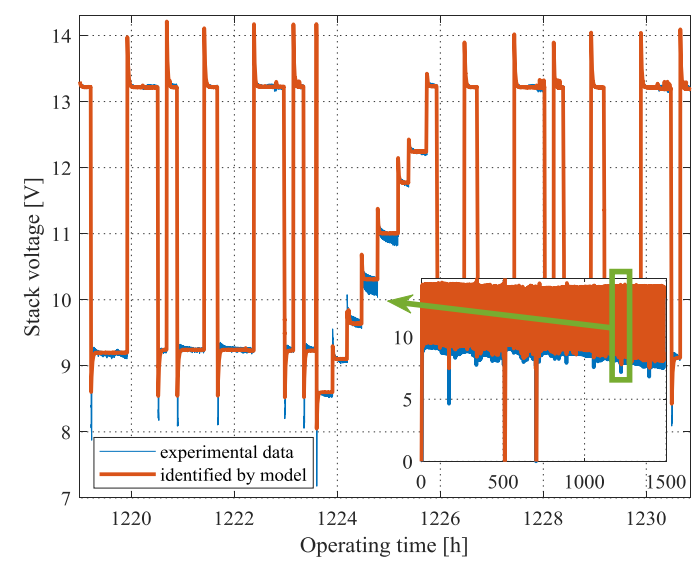

(a)

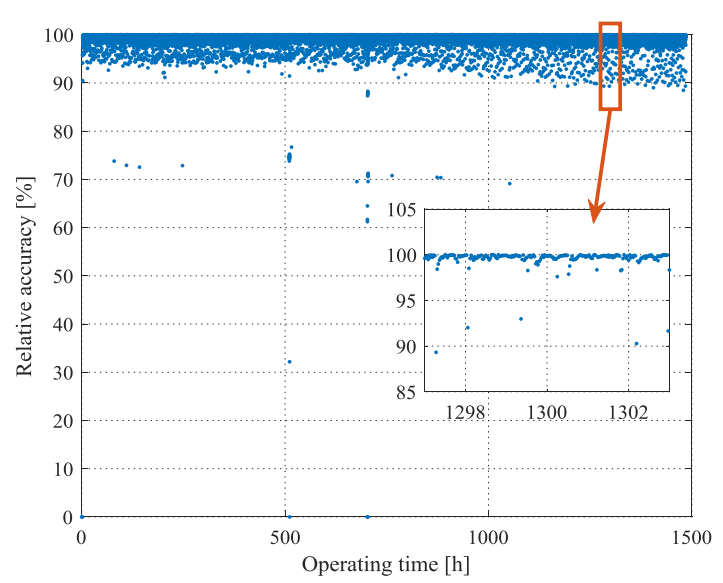

(b)

Fig. 11. Performance of the degradation behavior model: (a) identification of the stack voltage; (b) relative accuracy of the identification results.

Moreover, by bringing the above identified parameters into equation (25), the identified stack voltage $E_{\text {est }}$ can be obtained. Then the degradation behavior model is quantitatively evaluated using equation (26). Fig. 11 (a) shows the identification results of the stack voltage for all 1486 hours, with a zoom-in display of the results from hour 1219 to hour 1231. The results show that the model output voltage can identify the cyclical dynamic load changes and the operating condition long-term evolutions well. The proposed model can capture the voltage overshoot behavior in transition periods. Further, the model identification performance can be described quantitatively using $R A_{H I}$. As in Fig. 11 (b), $R A_{H I}$ is higher than $90 \%$ for almost all data points except for few outliers. In fact, the average $R A_{H I}$ calculated over all 1486 hours is $99.33 \%$. It is worth mentioning that the sampling rate of the aging experimental data is $1 \mathrm{~Hz}$, which means that the above average accuracy comes from millions of data.

\subsection{Performance of RUL estimation}

The second step in deploying the hybrid prognostic approach is the RUL estimation. As shown in Fig. 12 (a), considering the particularly unusual trend change in $\mathrm{HI}$ at point 1 , the first 122 hours $\mathrm{HI}$ should be ignored. A breakpoint is set every 74 hours (5\% of the total duration), and totally 10 breakpoints are set from hour 594 to hour 1262. The proposed hybrid prognostic approach is implemented at each breakpoint separately.

Specifically, the main parameters involved in ABBA are set as follows: $t o l=0.09, k=3, \omega_{\text {len }}=\omega_{\text {inc }}=0.5$. The three hyperparameters are set in a trial-and-error way. On the one aspect, the parameters should be assigned to achieve satisfactory ABBA representation. On the other aspect, the precision of RUL estimation should also be regarded. For 
the LSTM part, a random initial weight matrix to construct 20 different models, the learning rate is fixed at 0.001 , the maximum epoch is set to 100000, two LSTM layers and each contains 50 hidden units. Using the Adam optimizer [29], the training is automatically stopped when the training results reach the optimum. Intel ${ }^{\circledR}$ Xeon ${ }^{\circledR}$ E3-1230 v3 processor at 3.3GHz, 16 GB memory, Python 3.7.9, Keras version 2.3.1, and Tensorflow version 1.14.0 backend are respectively the hardware and software platforms. For comparison, Fig. 12 (b) and (c) show the prediction performance of ABBA-LSTM compared with Vanilla-LSTM with the same LSTM configuration at the hour 742 breakpoint. It is seen that the prediction results of Vanilla-LSTM hardly exceed the historical value range. In contrast, the features represented by ABBA effectively reduce the anomalous fluctuations while maintaining the degradation trend. Additionally, thanks to the symbolic-based representation, the training computing burden can be reduced. In our case, ABBA-LSTM takes 667.96 seconds while Vanilla-LSTM takes 768.43 seconds.

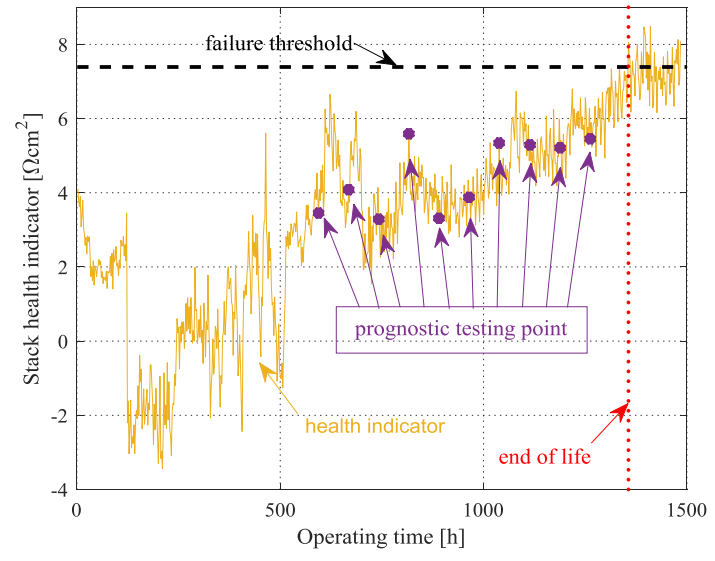

(a)

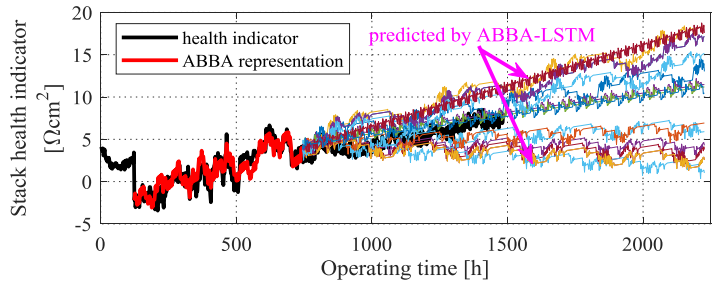

(b)

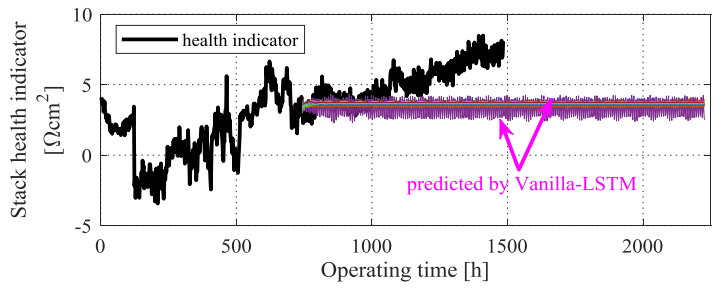

(c)

Fig. 12. Deployment of RUL estimation, (a) HI without outliers and breakpoints setting; (b) at hour 742, predicted degradation trends by ABBA-LSTM; (c) at hour 742, predicted degradation trends by Vanilla-LSTM.

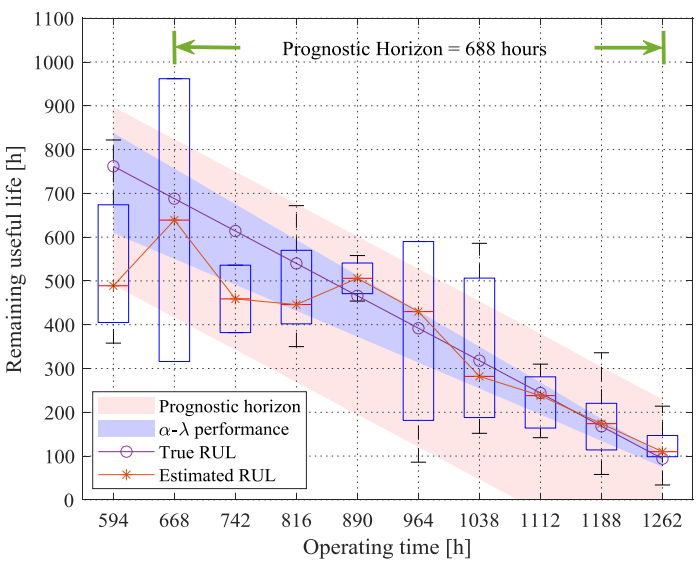

(a)

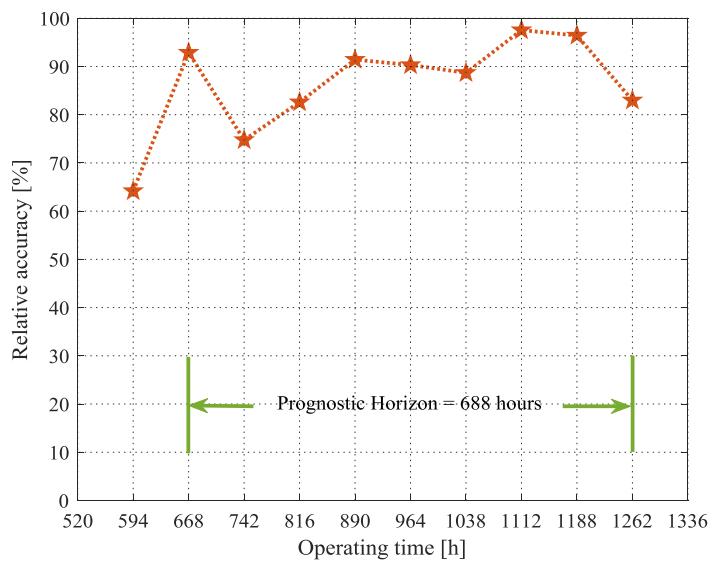

(b)

Fig. 13. RUL assessing: (a) prognostic horizon and $\alpha-\lambda$ performance; (b) relative accuracy.

In assessing the performance of the estimated RUL, the PH was first verified. As in Fig. 13 (a), the light pink zone represents the $A E Z_{P H}$. When the estimated RUL falls into this zone, it is considered a largely plausible result. Starting from hour 668, all subsequent results fall into $A E Z_{P H}$. The actual EOL is 1356 hours, and the $\mathrm{PH}$ can be calculated as 688 hours. Next, the light blue zone in Fig. 13 (a) represents $A E Z_{\alpha-\lambda}$, which is used to match with the estimated RUL in 
the PH interval. The results show that the estimated RUL is well matched, and only two outliers appear at hour 742 and hour 1262, as in Fig. 13 (b). Specifically, at hour 742, it is due to the abnormal shutdown of point 3 . And at hour 1262, it is due to a recoverable fault near hour 1225 . The average $R A_{R U L}$ in the $\mathrm{PH}$ interval is subsequently calculated as $88.62 \%$ using equation (30).

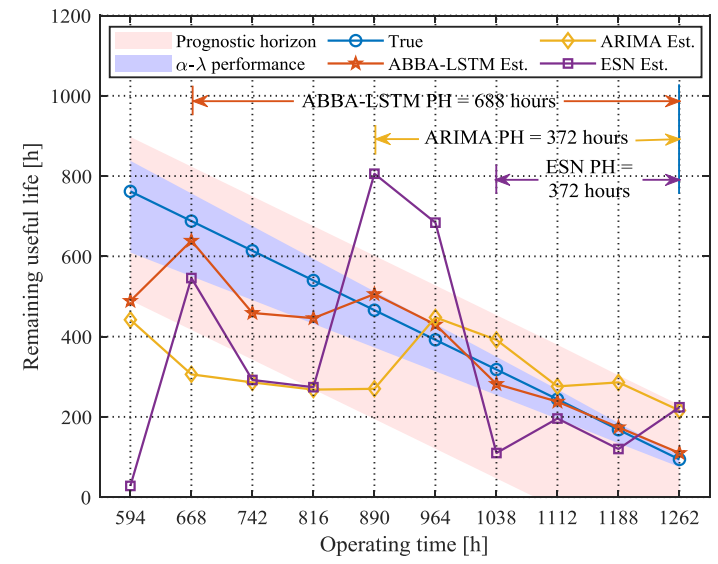

(a)

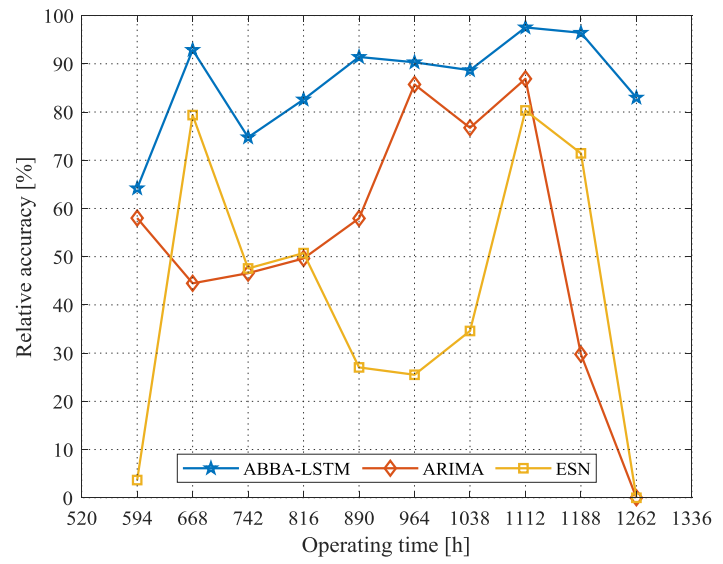

(b)

Fig. 14. Prognostic comparative results: (a) prognostic horizon and $\alpha-\lambda$ performance; (b) relative accuracy.

Further, as in Fig. 14, the proposed method was compared with the autoregressive integrated moving average model (ARIMA) and echo state network (ESN). In the comparison experiments, the input data of all three remain consistent with the previous, as in Fig. 12 (a). The comparative results show that the proposed method can provide longer PH. Within the respective PH interval, the proposed method achieves higher $R A_{R U L}$ and fits the $A E Z_{\alpha-\lambda}$ more closely. This also indicates that the proposed method has a credible long-term prognostic capability despite the lack of data pre-processing.

\subsection{Discussion}

The fuel cell prognostics in dynamic operating condition which represents one of the most difficult conditions is treated in this paper. More particularly, the prognostics is realized based only on the historical data of the concerned fuel cell stack. The prognostics model training and testing could be realized by using the data from a single long-term fuel cell test.

In this paper, a low-power stack with simple system design is focused on. The strategy could be generally applied to fuel cells of different power levels or current densities. However, it should be noted that fuel cells operated in different current density regions could demonstrate different dynamic behaviors coming from not only the fuel cell stacks, but also the auxiliary components and their control methods. In these conditions, the modified polarization function should be adjusted to the specific fuel cell system.

\section{Conclusion}

In this paper, a hybrid prognostic approach is proposed for estimating the RUL of PEMFC. A degradation behavior model is constructed and the health indicator associated with FC degradation is extracted through it. The degradation trend of health indicator is subsequently predicted with the help of ABBA-LSTM and RUL is estimated. The prognostic approach is evaluated using data from the FC aging experiment, and its performance can be summarized as 
follows.

- The proposed degradation model has the ability to identify the stack voltage dynamic behavior, and the average relative accuracy of the identification results reaches more than $99 \%$.

- The health indicator extracted by the proposed model shows a clear degradation trend. Also, the health indicator has potentially the ability to indicate different types of faults.

- The ABBA-LSTM is capable of capturing the trend information of health indicator and the prediction of the data in different data range can be achieved.

- The proposed approach provides a prognostic horizon approaching 50\% of the FC life-cycle, and the average relative accuracy of estimated RUL is close to $90 \%$.

The proposed approach has the ability to provide prognostic results with a wide horizon and high confidence, allowing more ample time for maintenance plan developments. However, it cannot be neglected that the degradation behavior hidden in FC systems is complex and difficult to be fully extracted. In the future work, efforts will be made to study FC intrinsic degradation phenomena at multiple scales and the influence of recovery faults.

\section{Acknowledgement}

This work was supported in part by the China Scholarship Council (CSC) under Grant [grant number 201906290107].

\section{References}

[1] McQueen S, Stanford J, Satyapal S, Miller E, Stetson N, Papageorgopoulos D, et al. Department of Energy Hydrogen Program Plan. Washington DC (United States): US Department of Energy (USDOE); 2020. https://doi.org/10.2172/1721803.

[2] Lohse-Busch H, Stutenberg K, Duoba M, Iliev S. Technology Assessment of a Fuel Cell Vehicle: 2017 Toyota Mirai. Argonne, IL (United States): Argonne National Lab. (ANL); 2018. https://doi.org/10.2172/1463251.

[3] Hart D, Jones S, Lewis J. The Fuel Cell Industry Review. E4tech 2020. https://fuelcellindustryreview.com/.

[4] Eudy L, Post MB. Fuel Cell Buses in U.S. Transit Fleets: Current Status 2018. Golden, CO (United States). National Renewable Energy Lab. (NREL) 2019. https://doi.org/10.2172/1489893.

[5] Sutharssan T, Montalvao D, Chen YK, Wang W-C, Pisac C, Elemara H. A review on prognostics and health monitoring of proton exchange membrane fuel cell. Renew Sustain Energy Rev 2017;75:440-50. https://doi.org/10.1016/j.rser.2016.11.009.

[6] Sharaf OZ, Orhan MF. An overview of fuel cell technology: Fundamentals andapplications. Renew Sustain Energy Rev 2014;32:810-53. https://doi.org/10.1016/j.rser.2014.01.012.

[7] Jouin M, Bressel M, Morando S, Gouriveau R, Hissel D, P'era M-C, et al. Estimating the end-of-life of pem fuel cells: guidelines and metrics. Appl Energy 2016;177:87-97. https://doi.org/10.1016/j.apenergy.2016.05.076.

[8] Jouin M, Gouriveau R, Hissel D, P'era M-C, Zerhouni N. Prognostics of Pem fuel cell in a particle filtering framework. Int J Hydrogen Energy 2014;39(1):481-94. https://doi.org/10.1016/j.ijhydene.2013.10.054.

[9] Lechartier E, Laffly E, P'era M-C, Gouriveau R, Hissel D, Zerhouni N. Proton exchange membrane fuel cell behavioral model suitable for prognostics. Int J Hydrogen Energy 2015;40(26):8384-97. https://doi.org/10.1016/j.ijhydene.2015.04.099.

[10] Ma R, Yang T, Breaz E, Li Z, Briois P, Gao F. Data-driven proton exchange membrane fuel cell degradation predication through deep learning method. Appl Energy 2018;231:102-15. https://doi.org/10.1016/j.apenergy.2018.09.111. 
[11] Wang C, Li Z, Outbib R, Zhao D, Dou M. Proton exchange membrane fuel cells prognostic strategy based on navigation sequence driven long short-term memory networks. IECON 2020 The 46th Annual Conference of the $\begin{array}{lllll}\text { IEEE Industrial } & \text { Electronics } & \text { 3969-3974. }\end{array}$ https://doi.org/10.1109/IECON43393.2020.9255373.

[12] Bressel M, Hilairet M, Hissel D, Bouamama BO. Extended Kalman filter for prognostic of proton exchange membrane fuel cell. Appl Energy 2016;164:220-7. https://doi.org/10.1016/j.apenergy.2015.11.071.

[13] Jouin M, Gouriveau R, Hissel D, P'era M-C, Zerhouni N. Degradations analysis and aging modeling for health assessment and prognostics of Pemfc. Reliab Eng Syst Saf 2016;148:78-95. https://doi.org/10.1016/j.ress.2015.12.003.

[14] Li Z, Zheng Z, Outbib R. Adaptive prognostic of fuel cells by implementing ensemble echo state networks in time-varying model space. IEEE Trans Ind Electron 2019;67(1):379-89. https://doi.org/10.1109/TIE.2019.2893827.

[15] Wang F-K, Mamo T, Cheng X-B. Bi-directional long short-term memory recurrent neural network with attention for stack voltage degradation from proton exchange membrane fuel cells. J Power Sources 2020;461:228170. https://doi.org/10.1016/j.jpowsour.2020.228170.

[16] Javed K, Gouriveau R, Zerhouni N, Hissel D. Prognostics of proton exchange membrane fuel cells stack using an ensemble of constraints based connectionist networks. J Power 2016;324:745-57. https://doi.org/10.1016/j.jpowsour.2016.05.092.

[17] Mezzi R, Yousfi-Steiner N, P'era MC, Hissel D, Larger L. An echo state network for fuel cell lifetime prediction under a dynamic micro-cogeneration load profile. Appl Energy 2021;283:116297. https://doi.org/10.1016/j.apenergy.2020.116297.

[18] Cheng Y, Zerhouni N, Lu C. A hybrid remaining useful life prognostic method for proton exchange membrane fuel cell. Int J Hydrogen Energy 2018;43(27):12314-27. https://doi.org/10.1016/j.ijhydene.2018.04.160.

[19] Liu H, Chen J, Hissel D, Su H. Remaining useful life estimation for proton exchange membrane fuel cells using a hybrid method. Appl Energy 2019;237:910-9. https://doi.org/10.1016/j.apenergy.2019.01.023.

[20] Elsworth S, Güttel S. Time series forecasting using 1stm networks: a symbolic approach. arXiv preprint arXiv:2003.05672, 2020. https://arxiv.org/abs/2003.05672.

[21] Jahnke T, Futter G, Latz A, Malkow T, Papakonstantinou G, Tsotridis G, et al. Performance and degradation of proton exchange membrane fuel cells: state of the art in modeling from atomistic to system scale. J Power Sources 2016;304:207-33. https://doi.org/10.1016/j.jpowsour.2015.11.041.

[22] Larminie J, Dicks A. Fuel cell systems explained. 2nd ed. Chichester: John Wiley \& Sons; 2003. https://doi.org/10.1002/9781118878330.

[23] Fletcher R. Practical methods of optimization. 2nd ed. Chichester: John Wiley \& Sons; 2000. https://doi.org/10.1002/9781118723203.

[24] Garnier H, Wang L. Identification of continuous-time models from sampled data. London: Springer; 2008. https://doi.org/10.1007/978-1-84800-161-9.

[25] Elsworth S, Güttel S. Abba: adaptive brownian bridge-based symbolic aggregation of time series. Data Min Knowl Disc 2020;34:1175-200. https://doi.org/10.1007/s10618-020-00689-6.

[26] Hochreiter S. The vanishing gradient problem during learning recurrent neural nets and problem solutions. Int J Uncertainty Fuzziness Knowledge Based Syst 1998;6(02):107-16. https://doi.org/10.1142/S0218488598000094.

[27] Hochreiter S, Schmidhuber J. Long short-term memory. Neural Comput 1997;9(8):1735-80. https://doi.org/10.1162/neco.1997.9.8.1735.

[28] Saxena A, Celaya J, Balaban E, Goebel K, Saha B, Saha S, et al. Metrics for evaluating performance of prognostic techniques. In: International conference on prognostics \& health management. IEEE; 2008. p. 1-17. https://doi.org/10.1109/PHM.2008.4711436.

[29] Kingma DP, Ba J. Adam: A Method for Stochastic Optimization. arXiv preprint arXiv:1412.6980, 2014. https://arxiv.org/abs/1412.6980. 